\title{
Glueless and Sutureless Multi-Layer Amniotic Membrane Transplantation in a Patient With Pending Corneal Perforation
}

\author{
Anastasios Lavaris ${ }^{1}$, Mohamed F M. Elanwar ${ }^{1}$, Motasim Al-Zyiadi ${ }^{1}$, Paraskevi T. Xanthopoulou ${ }^{1}$, Nick \\ Kopsachilis 1 \\ 1. Ophthalmology, East Kent Hospitals University NHS Foundation Trust, Canterbury, GBR
}

Corresponding author: Anastasios Lavaris, anastasios.lavaris@nhs.net

\begin{abstract}
Ocular graft-versus-host disease (GVHD) is a severe complication of allogenic hematopoietic stem cell transplantation (HSCT). It is a term used to describe a spectrum of signs and symptoms including ocular surface inflammation, dry eye syndrome, lacrimal and meibomian gland dysfunction. We present a case of a 73-year-old man with chronic myeloblastic leukaemia and chronic GVHD. On examination, severe corneal thinning was detected in his left eye. We performed multi-layer amniotic membrane patching of the affected area, in an ambulatory setting, without using sutures or glue, but only a bandage contact lens to keep amniotic membranes attached. Three months post-amniotic-membrane-patching symptoms improved, corneal integrity was maintained, and corneal thickness increased significantly. Multi-layer amniotic membrane patching without glue and sutures may be sufficient enough to prevent further deterioration of corneal thinning and can be safely performed as an outpatient procedure, reducing the need for tectonic corneal transplantation.
\end{abstract}

Review began $07 / 13 / 2021$ Review ended 07/19/2021 Published 07/27/2021

๑) Copyright 2021 Lavaris et al. This is an open access article distributed under the terms of the Creative Commons Attribution License CC-BY 4.0., which permits unrestricted use, distribution, and reproduction in any medium, provided the original author and source are credited.
Categories: Ophthalmology

Keywords: amniotic membrane, ocular graft-versus-host-disease, corneal thinning, ocular surface disease, corneal perforation

\section{Introduction}

Haematopoietic stem cell transplantation (HSCT), previously referred to as bone marrow transplantation, can be used in the treatment of immune haematologic disorders and haematologic malignancies. The source of donor cells can be the patient (autologous) or another individual (allogeneic) [1]. Despite the benefits of allogeneic HSCT in treating haematological malignancies, graft-versus-host disease (GVHD) is a serious post-transplantation complication. In GVHD, donor cells mount an immunologic attack against the host, mainly targeting the skin, gastrointestinal system, mouth, liver, lungs and eyes [2]. Ocular GVHD may affect up to $60 \%$ of patients receiving allogeneic HSCT and is mainly characterised by ocular surface and lacrimal glands inflammation and scarring, dry eye disease and meibomian gland dysfunction [3]. Dry eye syndrome (DES) is the most common ocular complication in patients undergoing HSCT [4]. Artificial tears, punctal plugs, topical corticosteroids and immunosuppressants are the main therapeutic options in treatment of ocular GVHD and associated DES [5]. The efficacy of amniotic membrane (AM) in treating severe immune or non-immune related DES, chemical and thermal injuries, Stevens-Johnson syndrome, immune-related peripheral ulcerative keratitis, necrotizing scleritis and limbal stem cell deficiency has been demonstrated. AM has anti-inflammatory, anti-microbial, anti-scarring, and anti-angiogenic effects when applied on the ocular surface. While sutured or glued AM patching has been used in treating corneal thinning and severe DES in cases of ocular GVHD, there are no reported cases in the literature of using a sutureless and glueless technique of multi-layer AM patching [5-8]; therefore we are presenting a case where this technique had a satisfying outcome.

\section{Case Presentation}

A 73-year-old man, with a known history of ocular graft-versus-host disease and a background of chronic myeloblastic leukaemia on oral ponatinib, who had undergone two bone marrow transplantations in the past (the latter being two years ago), was referred to our department by his optician. He was complaining of "exacerbation of his dry eye disease", with significant pain and photophobia in his left eye. His topical medication for dry eyes included preservative-free artificial tears used four times per day in both eyes and topical ciclosporin $1 \mathrm{mg} / \mathrm{ml}$ used twice per day in both eyes. His medication also included amlodipine and perindopril for arterial hypertension as well as oral zopiclone.

On examination, marked paracentral corneal stromal thinning was detected in his left eye (Figure 1). Neither infiltration nor anterior chamber reaction was present in either eye. Fundus examination was unremarkable in both eyes. 


\section{Cureus}

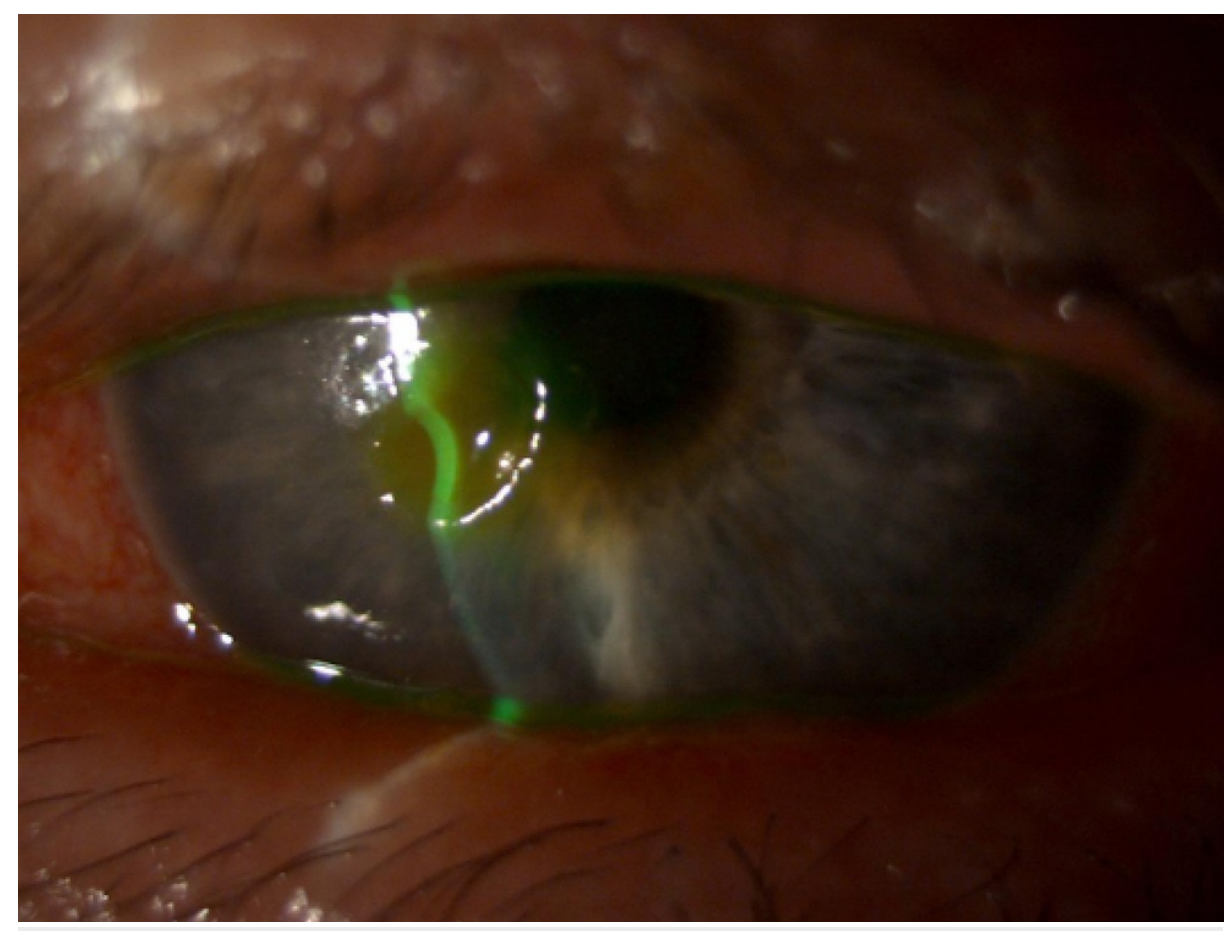

FIGURE 1: Slit lamp image of the left eye, prior to amniotic membrane transplantation, demonstrating marked paracentral stromal thinning

A sutureless and glueless technique of multi-layer amniotic membrane patching of the affected area was performed in his left eye, using a novel, low-temperature, vacuum-dried amniotic membrane (Omnigen ${ }^{\circledR}$; NuVision Biotherapies Ltd., Nottingham, UK). One amniotic membrane graft was folded and facilitated as material to fill the stromal thinning area and a second graft was used, stromal side down, to cover the first graft and the cornea. A large (20mm diameter bandage contact lens (Omnilenz ${ }^{\circledR}$; NuVision Biotherapies Ltd.) was fitted to the left eye. Following this procedure, a regimen of topical ciclosporin $1 \mathrm{mg} / \mathrm{ml}$, preservative-free dexamethasone $0.1 \%$, topical preservative-free chloramphenicol $0.5 \%$ and preservativefree cyclopentolate $1 \%$ was prescribed and follow up was arranged one week, one month, two months and three months following this procedure. Figures $2 a, 2 b, 2 c$ demonstrate anterior segment optical coherence tomography (OCT) image and corneal thickness measurement pre-operatively, at one month and two months following the procedure, respectively. One can see the increased thickness of the epithelial layer and the overall improved structural integrity of the cornea. 


\section{Cureus}

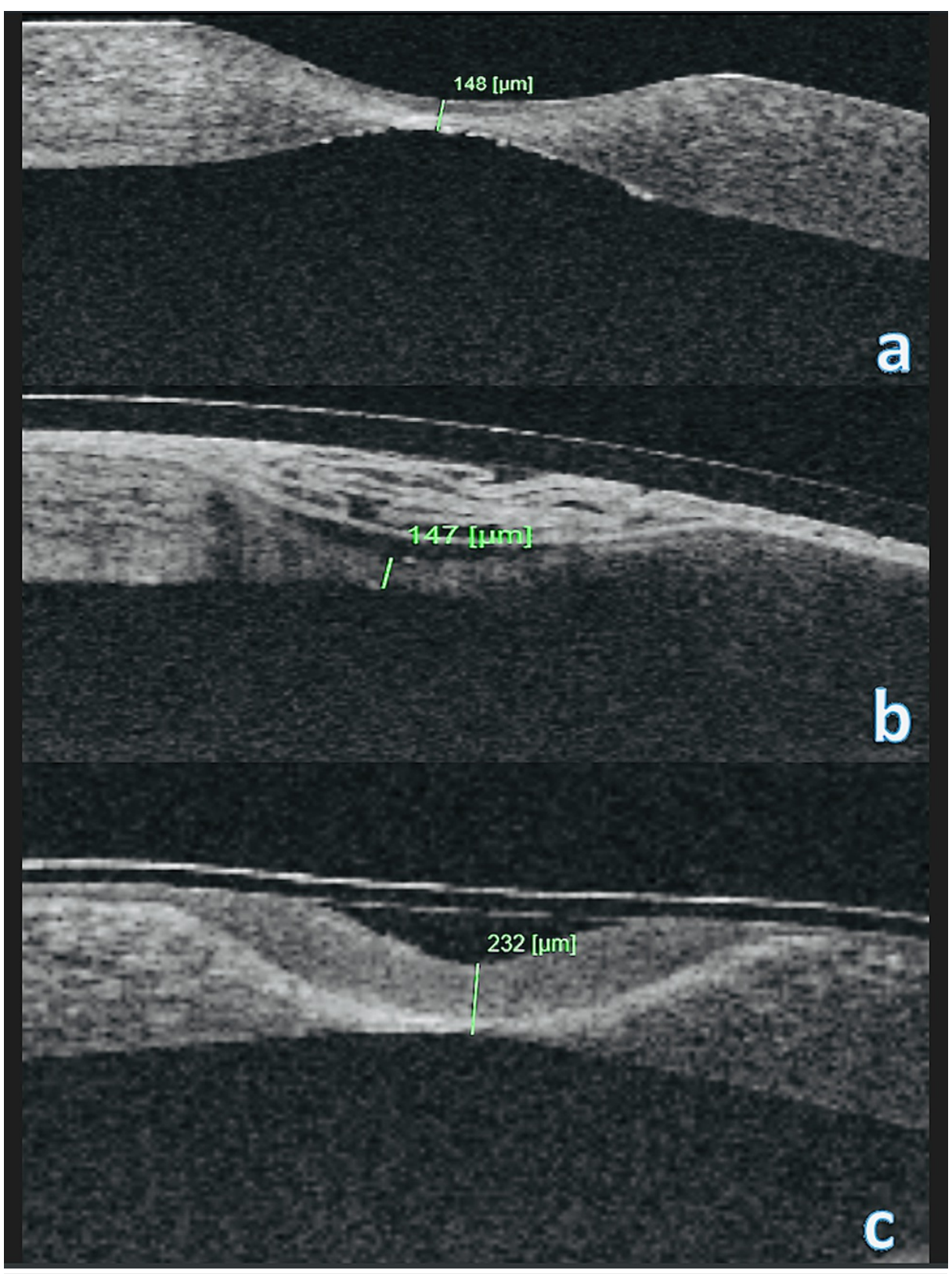

FIGURE 2: Anterior segment optical coherence tomography (OCT) and thickness measurement pre-operatively (a), at 1 month (b) and 2 months (c) following the procedure

\section{Discussion}

The armamentarium of available treatment options of DES and corneal thinning in ocular GVHD remains limited. Conventional treatment with tear supplements and bandage contact lenses is inadequate to reverse the immunologic process of GVHD and to prevent a corneal perforation.

Chronic use of topical corticosteroids has the known risks of corneal infection, cataractogenesis, ocular hypertension and glaucoma. Topical application of autologous serum drops may restore the ocular surface, but numerous disadvantages including reduced stability, risk of contamination and infections have been reported [9]. The application of sutured, cryopreserved AM in the management of ocular GVHD has been described $[6,10]$, but it has to be performed in an operating theatre setting.

\section{Conclusions}

In this regard, we underline the efficacy of sutureless and glueless vacuum-dried amniotic membrane application, as it can be easily performed in the clinic. The AM can integrate into the host cornea by formation of hemidesmosomes that provide anchoring for the regenerating epithelium. 
This outpatient clinic-based procedure, which eliminates the risk of surgical complications and sutureinduced inflammation, appears to be capable of preventing irreversible damage, including corneal perforation and the need for further surgical treatment.

\section{Additional Information \\ Disclosures}

Human subjects: Consent was obtained or waived by all participants in this study. Conflicts of interest: In compliance with the ICMJE uniform disclosure form, all authors declare the following: Payment/services info: All authors have declared that no financial support was received from any organization for the submitted work. Financial relationships: All authors have declared that they have no financial relationships at present or within the previous three years with any organizations that might have an interest in the submitted work. Other relationships: All authors have declared that there are no other relationships or activities that could appear to have influenced the submitted work.

\section{References}

1. Appelbaum FR: Hematopoietic-cell transplantation at 50. N Engl J Med. 2007, 357:1472-5. 10.1056/NEJMp078166

2. Schoemans HM, Lee SI, Ferrara JL, et al.: EBMT-NIH-CIBMTR Task Force position statement on standardized terminology \& guidance for graft-versus-host disease assessment. Bone Marrow Transplant. 2018, 53:1401-15. 10.1038/s41409-018-0204-7

3. Ogawa Y, Kim SK, Dana R, et al.: International Chronic Ocular Graft-vs-Host-Disease (GVHD) Consensus Group: proposed diagnostic criteria for chronic GVHD (Part I). Sci Rep. 2013, 3:3419. 10.1038/srep03419

4. Ogawa Y, Okamoto S, Wakui M, et al.: Dry eye after haematopoietic stem cell transplantation. Br J Ophthalmol. 1999, 83:1125-30. 10.1136/bjo.83.10.1125

5. Tung CI: Current approaches to treatment of ocular graft-versus-host disease . Int Ophthalmol Clin. 2017, 57:65-88. 10.1097/IIO.0000000000000167

6. Peris-Martínez C, Menezo JL, Díaz-Llopis M, Aviñó-Martínez JA, Navea-Tejerina A, Risueño-Reguillo P: Multilayer amniotic membrane transplantation in severe ocular graft versus host disease . Eur J Ophthalmol. 2001, 11:183-6. 10.1177/112067210101100215

7. Liang L, Sheha H, Li J, Tseng SC: Limbal stem cell transplantation: new progresses and challenges . Eye (Lond). 2009, 23:1946-53. 10.1038/eye.2008.379

8. Lazzaro DR: Repair of necrotizing scleritis in ulcerative colitis with processed pericardium and a Prokera amniotic membrane graft. Eye Contact Lens. 2010, 36:60-1. 10.1097/ICL.0b013e3181c6deb0

9. Ogawa Y, Okamoto S, Mori T, et al.: Autologous serum eye drops for the treatment of severe dry eye in patients with chronic graft-versus-host disease. Bone Marrow Transplant. 2003, 31:579-83. 10.1038/sj.bmt.1703862

10. Peric Z, Skegro I, Durakovic N, et al.: Amniotic membrane transplantation-a new approach to crossing the HLA barriers in the treatment of refractory ocular graft-versus-host disease. Bone Marrow Transplant. 2018, 53:1466-9. 10.1038/s41409-018-0140-6 